\title{
An educational program for decreasing catheter-related bloodstream infections in intensive care units: a pre- and post- intervention observational study
}

Yuichiro Shimoyama ${ }^{1 *}$, Osamu Umegaki ${ }^{1+}$, Tomoyuki Agui $^{2 \dagger}$, Noriko Kadono $^{1+}$, Nobuyasu Komasawa ${ }^{1+}$ and Toshiaki Minami ${ }^{1+}$

\begin{abstract}
Background: Central venous catheters (CVCs) are commonly used in the management of critically ill patients. This study aimed to determine whether an educational program could reduce the rate of catheter-related bloodstream infections (CRBSIs) in intensive care units (ICUs).

Findings: All patients admitted to a medical ICU at a college affiliated with the Japan Society of Intensive Care Medicine between January 2008 and December 2014 were surveyed prospectively for the development of CRBSIs. A mandatory educational program (the intervention) targeting an infection control committee consisting of physicians was developed by a multidisciplinary task force to highlight correct practices for preventing CRBSIs. The program included a 30-min video-based introduction, 120-min lectures with a number of hands-on training sessions, a post-test, posters, safety check sheets, and feedback from the infection control committee. Lectures based on the education program were held every 3 months, and participants were free to choose when they attended the lectures. Each participant was required to view the 30-min introduction before attending the 120-min lectures and complete the post-test after each lecture. Safety check sheets were made to ascertain adherence to contents of the educational program. Posters describing the educational program were posted throughout the ICU. A pre- and post-intervention observational study design was employed, with the main outcome measure being yearly CRBSIs. We also calculated cost savings that resulted from improved CRBSI rates. During the 12-month pre-intervention period, four episodes of CRBSIs occurred in 1171 patient ICU-days (i.e., 3.4 per 1000 patient ICU-days). In the first year after the intervention, the rate of CRBSIs decreased to 0 in 1157 patient ICU-days $(P \leq 0.05)$. The estimated cost savings secondary to this decreased rate for the 1 year following introduction of the program was between 1850,000 and $27,000,000$ yen $(\$ 14,800-\$ 216,000)$.
\end{abstract}

Conclusions: A program aimed at educating healthcare providers on the prevention of CRBSIs led to a dramatic decrease in the rate of primary bloodstream infections. This suggests that educational programs may substantially decrease medical care costs and patient morbidity attributed to central venous catheterization when implemented as part of mandatory training.

Keywords: Educational program, Catheter-related bloodstream infection, Intensive care unit

\footnotetext{
* Correspondence: shimocchiliebesfreud512@yahoo.co.jp

${ }^{\dagger}$ Equal contributors

'Department of Anesthesiology, Osaka Medical College, 2-7 Daigaku-machi,

Takatsuki, Osaka 569-8686, Japan

Full list of author information is available at the end of the article
} 


\section{Findings}

\section{Background}

Central venous catheters (CVCs) are commonly used in the management of critically ill patients. In the USA, 15 million CVC days (i.e., the total number of days of exposure to CVCs among all patients in a selected population during a selected time period) occur in intensive care units (ICUs) each year [1]. According to data regularly reported by the National Nosocomial Infection Surveillance (NNIS) system in the United States, more than $85 \%$ of primary bacteremias are catheter-related [2-4]. Catheter-related bloodstream infections (CRBSIs) cause considerable morbidity and mortality, and lead to high healthcare costs. Mortality attributable to CRBSIs has been estimated to be as high as $35 \%$, and length of hospital stay is consistently increased among infected patients in published reports $[5,6]$. Each year in the USA, CVCs cause an estimated 80,000 CRBSIs and, as a result, up to 28,000 deaths among patients in ICUs. These infections are associated with up to $\$ 2.3$ billion in annual costs [7].

To improve patient outcomes and reduce healthcare costs, there is considerable interest in reducing the incidence of these infections. Although there is no consensus on the optimal approach to achieve this, several recent studies indicate that educating healthcare providers on evidence-based approaches to prevent these infections can decrease infection rates [8-11]. The Centers for Disease Control and Prevention (CDC) recommends healthcare provider education as an important element of programs aimed at preventing hospital-acquired infections [12].

Against this backdrop, we carried out a clinical investigation to determine whether an educational program could reduce the rate of CRBSIs in the ICU of a teaching hospital. This study aimed to evaluate the effects of the program for up to 48 months after its implementation.

\section{Methods}

\section{Study location and patients}

This study was judged to not require registration by the institutional review board of Osaka Medical College (Osaka Japan) due to the use of unlinked anonymous data. This study was conducted in a medical ICU at a college affiliated with the Japan Society of Intensive Care Medicine. The medical ICU ( 8 beds) is an open unit with a multidisciplinary team providing patient care under the direction of attending physicians who are board certified in critical care medicine. Nurse staffing is maintained at a ratio of two patients per nurse. From January 2008, all patients admitted to the medical ICU were surveyed for the occurrence of CRBSIs. Intravascular catheters (e.g., CVCs, dialysis catheters, pulmonary artery catheters) used in the hospital were standard catheters without antimicrobial or antiseptic coatings. Arterial catheters were not surveyed in this study. The use of ultrasound/Doppler is recommended during insertions, except in emergent cases. Mostly residents (3-6 years of experience) and fellows (7-12 years of experience) insert CVCs at our hospital, and are required to use maximal sterile barrier precautions, including the use of a cap, mask, sterile gown, sterile gloves, and a sterile full body drape, when inserting CVCs. The primary study hypothesis was that the rate of CRBSIs would be reduced during the first year after implementation of the educational program (intervention) as compared with baseline. The secondary hypothesis was that the observed decrease in infection rate between 0 and 12 months after implementation of the program would be sustained over the subsequent observation period.

\section{Study design and data collection}

A pre- and post-intervention observational study design was employed with the main outcome measure being yearly CRBSIs. The educational program was developed in 2010 by an infection control committee comprising a physician, surgeon, anesthesiologist, intensivist, and nurses. The 2009 calendar year served as the baseline period for the incidence of CRBSIs. Throughout the study, data on the number of CRBSIs, as well as the following parameters, were recorded prospectively and on a daily basis: occurrence of primary bacteremia, number of patient ICU-days prior to the onset of bacteremia, and the species of microorganism associated with bloodstream infection. To coincide with the implementation period of the study intervention, daily data were aggregated into 1-year periods. The yearly rate of infection was calculated as the number of infections per 1000 patient-ICU days for each 1-year period. Yearly rates were assigned to one of six categories based on when the study intervention was implemented: at baseline, during the implementation period, or during one of four 1-year intervals up to four years after implementation. Data on who inserted the central catheters, average duration of catheter use, and number of lines in individual patients were not collected.

\section{Definitions}

A central catheter is defined herein as a catheter that ends at or near the heart or in a great vessel near the heart, and includes peripherally-inserted central catheters. CRBSIs were classified as primary or secondary based on CDC NNIS definitions [13]. Primary bloodstream infection (bacteremia) was defined using either of the following two criteria: (1) isolation of a recognized pathogen from blood culture (Staphylococcus aureus, Enterococcus species, Candida species) unrelated to 
infection at another site, and (2) fever of $\geq 38.0^{\circ} \mathrm{C}$, chills, or hypotension, and either of the following: common skin contaminant (e.g., Bacillus species, Propionibacterium species, coagulase-negative staphylococci, or micrococci) isolated from two blood cultures drawn on separate occasions within $24 \mathrm{~h}$ that are unrelated to infection at another site, or a common skin contaminant isolated from a blood culture of a patient with an intravascular device and the physician institutes appropriate antimicrobial therapy. CRBSI was defined as primary bacteremia in the presence of a CVC. Secondary bacteremia was defined as a bloodstream infection that develops as a result of a documented infection with the same microorganism at another body site.

\section{Intervention/educational program}

Newly hired physicians (interns, residents, fellows, attending physicians) were required to complete the educational program, which covered indications for intravascular catheter use, proper procedures for the insertion and maintenance of intravascular catheters, and appropriate infection control measures to prevent intravascular catheter-related infections. The program was implemented from April 2010 and consisted of a 30-min video introduction, 120-min lectures with a number of hands-on training sessions (not required for fellows and attending physicians given their experience), a post-test (not required for attending physicians given their experience), posters, safety check sheets, and feedback from the infection control committee. Lectures based on the education program were held every 3 months, and participants were free to choose when they attended the lectures. Contents of the safety check sheets were entered through each patient's computer terminal located directly outside the patient's room after inserting the CVC to improve clinician-to-medical safety committee communication within the hospital. This process allowed for monitoring adherence to the program's contents. The 120-min lectures and post-test were held every three months and based on CDC guidelines. The post-test was held after the 120-min lectures to reinforce the topic and group discussion of hands-on training. A score of $85 \%$ correct was required for the post-test. If this score was not achieved, the post-test was repeated until a passing score was achieved. Topics covered in the 120-min lectures and post-test included the epidemiology of CRBSIs, aseptic technique, use of maximal barrier precautions during central vein catheter insertion, preference for the jugular vein as a central vein insertion site, routine central vein catheter site care, proper technique for obtaining blood cultures, and guidelines for changing IV tubing and administration sets. The program was revised and recently updated in 2013 (Table 1). However, the updates were minor and the contents had not substantially changed
Table 1 Hospital guidelines for the prevention of catheterassociated bloodstream infection

1. Use a subclavian site, rather than a jugular or a femoral site, in adult patients to minimize infection risk for nontunneled CVC placement.

2. Avoid using the femoral vein for central venous access in adult patients.

3. Use a CVC with the minimum number of ports or lumens essential for the management of the patient.

4. Promptly remove any intravascular catheter that is no longer essential.

5. Perform hand hygiene procedures, either by washing hands with conventional soap and water or with alcohol-based hand rubs (ABHR). Hand hygiene should be performed before and after palpating catheter insertion sites as well as before and after inserting, replacing, accessing, repairing, or dressing an intravascular catheter.

6. Maintain aseptic technique for the insertion and care of intravascular catheters.

7. Use maximal sterile barrier precautions, including the use of a cap, mask, sterile gown, sterile gloves, and a sterile full body drape, for the insertion of CVCs, PICCs, or guidewire exchange.

8. Prepare clean skin with a $>0.5 \%$ chlorhexidine preparation with alcohol before central venous catheter insertion and during dressing changes.

9. Use either sterile gauze or sterile, transparent, semipermeable dressing to cover the catheter site.

10. Use a sterile sleeve for all pulmonary artery catheters.

11. Do not administer systemic antimicrobial prophylaxis routinely before insertion or during use of an intravascular catheter to prevent catheter colonization or CRBSI.

12. Do not routinely replace CVCs, PICCs, hemodialysis catheters, or pulmonary artery catheters to prevent catheter-related infections.

Abbreviation: CVC central venous catheter, PICC peripherally inserted central catheter, CRBSI catheter-related bloodstream infection

since 2010. Furthermore, item 1: "Use a subclavian site, rather than a jugular or a femoral site" is the general rule of our hospital. Limited to using in the operation room and ICU, it is stated clearly that a jugular site is the first choice by consideration of possibility of receiving positive pressure ventilation for patients. In addition to the educational program, a promotional campaign was launched beginning in April 2010 to augment the educational program. The campaign involved providing stickers on ID cards of staff members who completed the educational program, as well as posters describing the program which were displayed throughout the hospital.

\section{Blood culture technique}

Blood samples were obtained from two peripheral sites by physicians. Before collecting the blood sample, the skin was disinfected with $70 \%$ isopropyl alcohol and followed by $2 \%$ iodine tincture. When only one peripheral site was available and the patient had a central vein catheter in place, the second blood culture sample was obtained from the CVC [14]. Blood samples from CVCs 
were obtained from needleless caps that were disinfected with 70\% isopropyl alcohol, allowed to dry, and wiped with a povidone-iodine pad for $30 \mathrm{~s}$. Excess povidoneiodine was wiped off with sterile gauze prior to obtaining the sample. Three milliliters of blood were aspirated and discarded from both the CVC and peripheral venipuncture. A new syringe was used to aspirate an additional $20 \mathrm{~mL}$ of blood. A blood volume of $10 \mathrm{~mL}$ was injected into each of the two blood culture bottles. Injection of $\leq 5 \mathrm{~mL}$ of blood into a blood culture bottle was prevented in order to avoid false-negative results [15]. Attending physicians at the medical ICU and infection control staff at the hospital adjudicated contaminated cultures before submitting data.

\section{Cost analysis}

Numerous studies have attempted to quantify the costs associated with CRBSIs. The lowest of the published estimates comes from Arnow et al. [16], who estimated the cost to be $\$ 3700$ per infection using 1991 dollars, upon examining CVCs, arterial lines, and peripheral IV catheters in both floor and ICU patients. In more recent analyses, estimated costs associated with CRBSIs vary, ranging from $\$ 11,971$ to $\$ 54,000$ per episode of infection $[17,18]$. We calculated cost savings based on these previous studies in order to determine the impact of improved CRBSI rates resulting from the education program.

\section{Statistical analysis}

Statistical analysis was performed using SPSS 17.0 for Windows (SPSS Inc., Chicago, IL, USA). Fisher's exact test was used to compare categorical variables. The incidence rate of CRBSIs per 1000 patient ICU-days was calculated, and the risk difference of CRBSIs in the postvs pre-intervention period was determined. All reported $P$ values are two-sided. $P \leq 0.05$ was considered statistically significant.

\section{Results}

During the 12-month pre-intervention period (calendar year 2009), a total of four episodes of CRBSI occurred during a total of 1171 patient ICU-days. This amounts to an infection rate of 3.4 per 1000 patient ICU-days. During the post-intervention period (calendar year 2011), no episodes of CRBSI were recorded for a total of 1157 patient ICU-days. The rate of CRBSIs decreased from 3.4 infections per 1000 patient ICU-days at baseline to 0 at $0-12$ months after implementation of the program $(P \leq 0.05)$. The difference in CRBSI rates between the preand post-intervention periods was 3.4 per 1000 catheter ICU-days. However, the markedly reduced rates of CRBSI were not sustained for up to 36 months of follow-up (Table 2).

\section{Cost analysis}

Assuming a continued infection rate of 3.4 per 1000 patient ICU-days (pre-intervention rate), four infections would have been expected to occur for the 1157 patient ICU-days in the 12 months after the intervention. When the reduced number of CRBSI occurrences (total of four) resulting from the educational program was calculated, the savings associated with improvements ranged from $¥ 1,850,000$ to $¥ 27,000,000$ ( $\$ 14,800-\$ 216,000$ ) annually using the yen-dollar exchange rate in 2015 .

\section{Discussion}

This study demonstrated that an educational program for physicians working at Osaka Medical College was able to significantly reduce the incidence of CRBSIs. These results suggest that behavioral changes may have played a crucial role in the success of the program. Simulation-based learning was recently found to be more effective than video training alone in improving resident skills [19]. In the context of the present study, simulation-based learning substantially reduced the CRBSI rate relative to the control group. Overall, our findings suggest that an education-based intervention aimed at optimizing CVC care could be successfully implemented in a teaching hospital and have significant public health consequences. Importantly, the intervention can be implemented without the need for expensive technology or additional ICU staffing.

Our results are consistent with those of published studies reporting significant reductions in the occurrence of hospital-associated infections with the implementation of education-based interventions. For instance, previous studies have reported $66,58.5$, and $27 \%$ reductions in the incidence of CRBSIs as a result of implementing education-based programs [8, 20, 21]. Education-based programs have previously reduced ventilator-associated pneumonia (57.6\% decrease in infections in five ICUs) [10]. Use of the same educational program in a pediatric hospital and a community hospital had similar results [11]. In one study, an educational program was associated with a $53.6 \%$ reduction in patient colonization of vancomycin-resistant enterococci [22]. These results and published studies support the following recommendations of the CDC: "Educate healthcare personnel regarding the indications for intravascular catheter use, proper procedures for the insertion and maintenance of intravascular catheters, and appropriate infection control measures to prevent intravascular catheter-related infections" (Category IA of the Guidelines for the Prevention of Intravascular Catheter-related Infections, 2011 [23]).

Within a year after implementation, the rate of CRBSIs was reduced to 0 . However, this significant reduction was not sustained over a longer period (i.e., throughout 
Table 2 Baseline data

\begin{tabular}{|c|c|c|c|c|c|c|}
\hline Study period (year) & $\mathrm{N}$ & No. of infections & Patient ICU-days & $\begin{array}{l}\text { No. of infections per } 1000 \\
\text { patient ICU-days }\end{array}$ & Incidence-rate ratio & $P$ value \\
\hline Baseline (2009) & 176 & 4 & 1171 & 3.4 & 1 & \\
\hline Begin implementation (2010) & 172 & 0 & 1189 & 0 & - & \\
\hline \multicolumn{7}{|l|}{ After implementation } \\
\hline 1 year (2011) & 195 & $0 *$ & 1157 & 0 & - & 0.0497 \\
\hline 2 years (2012) & 191 & 1 & 1722 & 0.58 & 0.11 & 0.1985 \\
\hline 3 years (2013) & 171 & 1 & 1438 & 0.7 & 0.14 & 0.3716 \\
\hline 4 years (2014) & 207 & 1 & 1598 & 0.68 & 0.13 & 0.1845 \\
\hline
\end{tabular}

$\circledast_{p}$ value $<0.05$ was considered statistically significant

Abbreviation; ICU intensive care unit

3 years of follow-up; Table 2). This result suggests that beneficial effects of the intervention may not persist for long periods. What can we do to maintain the beneficial effects over a longer period? An important common element underlying the success of the program was the wide exposure of the program to hospital staff who directly care for patients consecutively. The effects of these educational programs may be even more pronounced in the longer-term if these hospital staff are involved in designing the specific measures taught in the program. In the context of this study, the educational program targeted the infection control committee, which consisted of physicians. This should be purposefully done in order to impact healthcare providers most closely involved in performing the interventions described in the program.

CRBSI rates greater than 1 or 2 per 1000 patient ICUdays are no longer acceptable in current medical settings [24]. The consistent impact of educational programs on the reduction of CRBSIs suggests that their implementation should be routine in hospital environments where patients are at risk of being infected. Efforts to increase compliance with the established protocols should be discussed and updated on a regular basis, and continuous surveillance of CVC infection rates with feedback to staff should be implemented. We suggest that educational programs be renewed/updated in intervals of several years and making participation in the programs mandatory for all new employees.

Despite our positive outcomes, there are a number of limitations worth noting. First, the study was conducted in an ICU of a single hospital, so the results may not be broadly generalizable. Second, we did not collect data based on the positions of hospital staff (e.g., interns, residents, fellows, attending physicians) who inserted the central catheters, or the varying experiences of attending physicians versus interns, for example, regarding CVC insertion. This raises the possibility that differences in behavior/experience other than the implementation of the educational program may have accounted for these results. Third, we did not record the duration of central venous catheterization or the number of lines in individual patients. An unrecognized reduction in the duration of catheterization or the number of lines could have influenced our results. Fourth, our study design does not allow us to determine which aspects of the intervention accounted for the reduction in the rate of CRBSIs. Another potential limitation is that we did not evaluate outcomes other than bloodstream infection. As a result, we cannot determine whether the intervention influenced antibiotic utilization, length of hospital stay, mortality, or antibiotic resistance patterns.

Despite these limitations, our results indicate that implementation of the educational program directly or indirectly reduced the rate of CRBSIs. Thus, we were able to achieve the original aim of the study, despite the limitations discussed above and inability to identify the specific aspects of the intervention accounting for the reduction.

\section{Conclusions}

Here, we demonstrated that a relatively simple educational program can reduce CRBSIs in a hospital setting. The observed reduction in rate from 3.4 per 1000 patient-ICU days to 0 per 1,000 patient-ICU days was associated with an estimated cost savings of between $¥ 1,850,000$ and $¥ 27,000,000$ annually within the first year after implementing the program. However, rates did not remain significantly reduced throughout the remaining three years of follow-up. Although the program needs further refinement, our findings suggest that the implementation of education-based infection control programs is important for the optimal functioning of medical ICUs.

\section{Abbreviations}

CDC: The Centers for Disease Control and Prevention; CRBSI: Catheter-related bloodstream infection; CVC: Central venous catheter; ICU: Intensive care units; NNIS: National Nosocomial Infection Surveillance

Acknowledgements

None. 


\section{Funding}

Research funding was provided by the Department of Anesthesiology, Osaka Medical College.

\section{Availability of data and materials}

We do not wish to share our raw data, which were submitted to Ministry of Health, Labour and Welfare, as we do not have the copyright.

\section{Authors' contributions}

YS participated in the study design, collected data, performed the statistical analysis, and drafted the manuscript; OU, TA, NK, NK, and TM participated in the study design and revised the manuscript. All authors read and approved the final manuscript.

\section{Authors' information}

None.

\section{Competing interests}

The authors declare that they have no competing interests.

\section{Consent for publication}

Not applicable.

\section{Ethics approval and consent to participate}

Not applicable.

\section{Publisher's Note}

Springer Nature remains neutral with regard to jurisdictional claims in published maps and institutional affiliations.

\section{Author details}

'Department of Anesthesiology, Osaka Medical College, 2-7 Daigaku-machi, Takatsuki, Osaka 569-8686, Japan. ²Department of Surgery, Osaka Medical College, Takatsuki, Japan.

Received: 13 January 2017 Accepted: 2 May 2017

Published online: 08 May 2017

\section{References}

1. Mermel LA. Prevention of intravascular catheter-related infections. (Erratum: Ann Intern Med 133:395, 2000). Ann Intern Med. 2000;132:391-402.

2. Richards MJ, Edwards JR, Culver DH, Gaynes RP. Nosocomial infections in coronary care units in the United States. National Nosocomial Infections Surveillance System. Am J Cardiol. 1998;82:789-93.

3. Richards MJ, Edwards JR, Culver DH, Gaynes RP. Nosocomial infections in pediatric intensive care units in the United States. National Nosocomial Infections Surveillance System. Pediatrics. 1999;103:39-45.

4. Richards MJ, Edwards JR, Culver DH, Gaynes RP. Nosocomial infections in medical intensive care units in the United States. National Nosocomial Infections Surveillance System. Crit Care Med. 1999;27:887-92.

5. Dimick JB, Pelz RK, Consunji R, Swoboda SM, Hendrix CW, Lipsett PA. Increased resource use associated with catheter-related bloodstream infection in the surgical intensive care unit. Arch Surg. 2001;136:229-34.

6. Pittet D, Tarara D, Wenzel RP. Nosocomial bloodstream infection in critically ill patients: excess length of stay, extra costs, and attributable mortality. JAMA. 1994;271:1598-601.

7. Klevens RM, Edwards JR, Richards Jr CL, Horan TC, Gaynes RP, Pollock DA, et al. Estimating health care-associated infections and deaths in US hospitals, 2002. Public Health Rep. 2007;122:160-6.

8. Coopersmith CM, Rebmann TL, Zack JE, Ward MR, Corcoran RM, Schallom $M E$, et al. Effect of an education program on decreasing catheter-related bloodstream infections in the surgical intensive care unit. Crit Care Med. 2002;30:59-64.

9. Warren DK, Zack JE, Cox MJ, Cohen MM, Fraser VJ. An educational intervention to prevent catheter-associated bloodstream infections in a nonteaching, community medical center. Crit Care Med. 2003;31:1959-63.

10. Zack JE, Garrison T, Trovillion E, Clinkscale D, Coopersmith CM, Fraser VJ, et al. Effect of an education program aimed at reducing the occurrence of ventilator-associated pneumonia. Crit Care Med. 2002;30:2407-12.
11. Babcock HM, Zack JE, Garrison T, Trovillion E, Jones M, Fraser VJ, et al. An educational intervention to reduce ventilator-associated pneumonia in an integrated health system: a comparison of effects. Chest. 2004;125:2224-31.

12. Healthcare Infection Control Practices Advisory Committee; Centers for Disease Control and Prevention (U.S.). Guidelines for preventing health-careassociated pneumonia, 2003 recommendations of the CDC and the Healthcare Infection Control Practices Advisory Committee. Respir Care. 2004:49:926-39.

13. National Nosocomial Infections Surveillance (NNIS) system report: data summary from October 1986-April 1998, issued June 1998. Am J Infect Control. 1998:26:522-33.

14. Beutz M, Sherman G, Mayfield J, Fraser VJ, Kollef MH. Clinical utility of blood cultures drawn from central vein catheters and peripheral venipuncture in critically ill medical patients. Chest. 2003;123:854-61.

15. Weinstein MP, Mirrett S, Wilson ML, Reimer LG, Reller LB. Controlled evaluation of 5 versus 10 milliliters of blood cultured in aerobic BacT/Alert blood culture bottles. J Clin Microbiol. 1994;32:2103-06.

16. Arnow PM, Quimosing EM, Beach M. Consequences of intravascular catheter sepsis. Clin Infect Dis. 1993;16:778-84.

17. O'Grady NP, Alexander M, Dellinger EP, Gerberding JL, Heard SO, Maki DG, et al. Guidelines for the prevention of intravascular catheter-related infections. Centers for Disease Control and Prevention. MMWR Recomm Rep. 2002;51:1-29.

18. Warren DK, Quadir WW, Hollenbeak CS, Elward AM, Cox MJ, Fraser VJ. Attributable cost of catheter-associated bloodstream infections among intensive care patients in a nonteaching hospital. Crit Care Med. 2006;34: 2084-9.

19. Khouli H, Jahnes K, Shapiro J, Rose K, Mathew J, Gohil A, et al. Performance of medical residents in sterile techniques during central vein catheterization: randomized trial of efficacy of simulation-based training. Chest. 2011;139:80-7.

20. Warren DK, Zack JE, Mayfield JL, Chen A, Prentice D, Fraser VJ, et al. The effect of an education program on the incidence of CVC-associated bloodstream infection in a medical ICU. Chest. 2004:126:1612-18.

21. Sherertz RJ, Ely EW, Westbrook DM, Gledhill KS, Streed SA, Kiger B, et al. Education of physicians-in-training can decrease the risk for vascular catheter infection. Ann Intern Med. 2000;132:641-8.

22. Puzniak LA, Leet T, Mayfield J, Kollef M, Mundy LM. To gown or not to gown: the effect on acquisition of vancomycin-resistant enterococci. Clin Infect Dis. 2002;35:18-25.

23. O'Grady NP, Alexander M, Burns LA, Dellinger EP, Garland J, Heard SO, et al. Healthcare Infection Control Practices Advisory Committee. Guidelines for the prevention of intravascular catheter-related infections. Clin Infect Dis. 2011;52:162-93.

24. Timsit JF, Dubois Y, Minet C, Bonadona A, Lugosi M, Ara-Somohano C, et al. New materials and devices for preventing catheter-related infections. Ann Intensive Care. 2011;1:34

\section{Submit your manuscript to a SpringerOpen ${ }^{\circ}$ journal and benefit from:}

- Convenient online submission

- Rigorous peer review

- Immediate publication on acceptance

- Open access: articles freely available online

- High visibility within the field

- Retaining the copyright to your article

Submit your next manuscript at $>$ springeropen.com 